\title{
The imaging and cognition genetics conference 2011, ICG 2011: a meeting of minds
}

\author{
Stéphanie Le Hellard ${ }^{1,2,3 *}$ and Isabel Hanson ${ }^{4}$ \\ ${ }^{1}$ Centre for Advanced Study, Oslo, Norway \\ ${ }^{2}$ Dr E. Martens Research Group for Biological Psychiatry, Department of Clinical Medicine, University of Bergen, Bergen, Norway \\ ${ }^{3}$ Center for Medical Genetics and Molecular Medicine, Haukeland University Hospital, Bergen, Norway \\ ${ }^{4}$ Isabel Hanson Scientific Writing, Edinburgh, UK
}

\section{Edited by:}

Robert W. Williams, University of Tennessee Health Science Center, USA

\section{Reviewed by:}

Detlef H. Heck, University of Tennessee Health Science Center, USA

Ruth Siegel, Case Western Reserve University, USA

\section{${ }^{*}$ Correspondence:}

Stéphanie Le Hellard, Section for Medical Genetics and Molecular Medicine, Haukeland University Hospital, Laboratory Building, 6th floor, N-5021 Bergen, Norway. e-mail: stephanie.hellard@med.uib.no
In June 2011, 70 researchers from the disciplines of cognitive science, genetics, psychology, psychiatry, neurobiology, and computer science gathered in Os, Norway, for the first Imaging and Cognition Genetics meeting. The aim of the conference was to discuss progress, enhance collaboration, and maximize the sharing of resources within this new field. In this Perspective, we summarize the major themes that emerged from ICG 2011. The first is the importance of defining cognitive and imaging phenotypes and endophenotypes suitable for genetic analysis. These can come from differential psychology, cognitive science, structural MRI, tractography, and functional imaging. The second theme is the emergence of new methods for the analysis of complex traits. These include advanced computational and statistical techniques for analyzing complex datasets, and new ways of interpreting data from genome-wide association studies, such as jointly evaluating the contribution of SNPs in specific genes and pathways rather than considering single SNPs in isolation. The final theme is the importance of establishing functional correlates of newly identified genetic variants.

Keywords: imaging, cognition, genetics, neurogenetics, GWAS

\section{INTRODUCTION}

Understanding changes in cognitive ability, in relation to healthy aging and the onset of dementia or psychiatric disorders, is a major research priority. Large samples of individuals with cognitive and imaging phenotyping data are now available, and genetic data have been collected for many. To analyze these samples effectively, it is necessary to establish the basic principles which will ensure that the power of modern imaging techniques and statistical genetic methods are used to maximum advantage. It is crucial for the new field of imaging and cognition genetics that lessons are learned from other areas of complex trait analysis, so that future studies have the best possible design. This is the background to ICG 2011. Here we summarize the three main themes that emerged from the meeting.

\section{IDENTIFICATION OF TRAITS SUITABLE FOR GENETIC ANALYSIS}

A special emphasis of ICG 2011 was on identifying cognitive and imaging traits that can be dissected genetically. Suitable phenotypes and endophenotypes should show heritable variation in the population, and must be measurable in an accurate and reproducible way. Even highly complex neurocognitive phenotypes like intelligence, attention, and memory have components that can be reliably measured.

Ian Deary reviewed the differential psychology approach to investigating intelligence. Human mental capabilities can be described by a hierarchy with a general intelligence factor $(g)$ at the top, which contributes to more specialized cognitive domains in the middle, which in turn contribute to ability in specific tests at the lowest level (Deary et al., 2010). Finding the factors that underlie differences in human intelligence depends on examining all three levels of cognitive function. The genetic effect is complex, with some contributions to overall cognitive ability and some to specific cognitive domains or skills.

Thomas Espeseth described tests of attentional function in the Norwegian Cognitive NeuroGenetics (NCNG) sample. Individuals homozygous for the T-allele of a SNP in the nicotinic receptor alpha 4 subunit gene performed better at high load for attention tasks (Espeseth et al., 2007, 2010). In a visual attention test, T carriers had a higher rate of visual short-term memory encoding than non-T carriers. These results were replicated using the same task in a cohort from George Mason University. This and other ongoing replications of NCNG association data in the GMU sample are rare examples of direct task-to-task comparisons that can lead to successful genetic replications.

Yulia Kovas presented an investigation of mathematical ability in the Twins Early Development Study (7,500 pairs of UK twins aged 2-16, assessed for development, behavior, personality, and educational attainment). Variation in mathematical ability is highly heritable and is probably due to multiple loci of small effect. A genome-wide association studies (GWAS) performed on pooled DNA from children of low and high mathematical ability (validated in a larger study; Docherty et al., 2010) revealed a set of 10 SNPs that explains about $3 \%$ of the phenotypic variance and is also related to non-mathematical cognitive abilities. There is evidence 
of an environmental effect on genotype (Docherty et al., 2011).

Andreas Papassotiropoulos explained how work on memory and synaptic plasticity in animals has yielded numerous candidates for studies of the genetic basis of inter-individual variability in human memory. Examples include HTR2A, the human serotonin $2 \mathrm{~A}$ receptor gene and $A D R A 2 B$, encoding the alpha- $2 \mathrm{~b}$ adrenergic receptor (Rasch et al., 2009). A screen of SNPs in 47 genes implicated in memory by animal studies revealed a profile of genetic variability linked to performance in episodic memory tasks and activation of brain regions involved in memory (de Quervain et al., 2007). However, much of the estimated heritability of memory is still unaccounted for, and may reside in rare variants, epigenetic effects, and gene/gene or gene/environment interactions. A preliminary test for gene/gene interactions focused on SNPs in human homologs of genes that are differentially expressed in rat hippocampus during a water maze-based learning test. Papassotiropoulos emphasized the importance when carrying out association tests of the phenotype under study - episodic memory can be measured in many different ways which may depend on different encoding mechanisms, especially if the test has an emotional component. The ultimate goal of these studies is to translate genetic findings into clinical treatments, and clinical trials are underway to test drugs that could affect genes identified by previous association studies (e.g., KIBRA) or by the latest GWAS.

Turning to psychiatric disorders, Kenneth Hugdahl described a "constrained phenotype" approach to studying schizophrenia, focusing on one common symptom, auditory hallucinations. These are typically negative, uncontrollable voices which appear to be of external origin. Simple auditory tests revealed that auditory processing is abnormal in schizophrenia patients (Hugdahl, 2009). Meta-analysis of fMRI data showed that in patients the auditory cortex is spontaneously activated during auditory hallucinations, but under-activated by external sounds. Healthy individuals can use attentional focus to distinguish between different sounds presented simultaneously in auditory tests, but patients with frequent auditory hallucinations lack this "top-down" control (Hugdahl et al., 2009). Abnormal neural activity in the temporal regions of the brain seems to cause mis-representation of internally generated stimuli, which appear to have an external source. This misattribution is maintained through deficient inhibitory functions in frontal and parietal regions (Hugdahl, 2009). Analyzing the mechanisms that underlie neural hyper-excitation could lead to better molecular and genetic understanding of auditory hallucinations and psychiatric disorders.

Brain morphometry is another important source of phenotypes. Anders Fjell described the role of structural imaging in understanding age-related brain changes. By standardizing segmentation techniques, data from different samples obtained with different scanners can be pooled. This shows that cortical thinning is most pronounced in the superior and inferior frontal gyri and superior temporal cortices (Fjell et al., 2009). Cortical thinning results from reduced numbers of synapses and dendritic spines and shrinkage of neuronal bodies. Cortical volume is highly heritable, as are cortical area and cortical thickness, but these phenotypes may be genetically independent of each other. In a twin study, the heritability of white matter integrity depended on factors including age, IQ, and socio-economic status (Chiang et al., 2011).

Lars Nyberg presented fMRI studies of the Betula Prospective Cohort Study of aging, memory, and health. Functional MRI scanning of an abstract categorization task which engages the frontal and parietal regions revealed that APOE4 was associated with dose-dependent reduction in activation of the left inferior parietal cortex and bilateral anterior cingulate (Lind et al., 2006). APOE4 carriers also had reduced hippocampal volume and lower white matter integrity in the corpus callosum (Persson et al., 2006). Nyberg also reported that depending on COMT genotype, different parts of the brain had to work harder in response to different functional elements of a working memory test. A large-scale imaging genetics study of over 350 Betula subjects is underway, involving fMRI of several memory tasks to engage specific brain regions. The team is investigating whether a "brain score," suitable for use in GWAS, can be calculated for each subject based on the weighted average of voxels contributing to the activation pattern for a given task.

Lorna Lopez described how a "white mater integrity factor" (Penke et al., 2010) had been calculated by DTI of 16 white matter tracts in 672 healthy aged individuals from the Lothian Birth Cohort 1936. Following a GWAS of white matter integrity, no genome-wide association at the single SNP level was identified. Two gene-based methods, LDsnpR (Christoforou et al., 2012) and VEGAS, yielded no genes passing the correction for multiple testing, but both gene-based lists were enriched for genes involved in synapse function.

\section{ANALYSIS OF COMPLEX TRAITS: CURRENT INVESTIGATIONS AND NEW METHODS}

Recent studies have shown that the genetic analysis of complex traits such as psychiatric disorders and intelligence will require new tools to better tackle the high polygenicity and heterogeneity of these traits. Several presentations focused on such approaches.

Gail Davies presented GWAS studies of fluid $g$ (Gf) and crystallized $g(\mathrm{Gc})$ in the CAGES cohorts (Cognitive Aging in England and Scotland). No SNP reached genome-wide significance, but the QQ plots strongly indicated a polygenic effect. The estimated proportion of the phenotype variance explained by the variance in the SNPs tested was 40\% for Gc and 51\% for Gf (Davies et al., 2011). Gf and Gc appear to be highly polygenic, meaning that extremely large cohorts will be needed to identify the underlying variants by GWAS.

Andrea Christoforou described gene- and pathway-based methods for analyzing an ongoing GWAS of Gc and Gf in 670 subjects from the NCNG cohort. At the single SNP level there was no genome-wide significant association, but when the most strongly associated SNPs were tested as candidate sets by set enrichment analysis (GSEA) in the CAGES cohorts, the Gc SNP-set was significantly enriched. Pairwise SNP-SNP interactions, tested using the InterSNP program (Herold et al., 2009), yielded one significant interaction for Gf and several for Gc. Gene-level analysis, performed with LDsnpR (Christoforou et al., 2012) and gene ontology, gave no significant hits for Gf, but Gc showed significant association with genes involved in neuronal 
differentiation and axon guidance. Replication in the CAGES cohort was seen for Gc at the individual gene and gene set levels.

Continuing on the theme of gene sets, Kari Ersland described how genes that are differentially expressed in the frontomedial, occipital, and temporal regions of the adult rat cortex (Stansberg et al., 2011) can be used as candidate gene sets for enrichment tests against four cognitive domains, and against bipolar affective disorder (BPAD) and schizophrenia. The temporal cortex gene set was significantly enriched in the gene list generated from a matrix reasoning task.

Andrew Brown described a competitive pathway test for studying genetic control of brain function. All the SNPs in the genes in a given pathway are examined to see if they are better represented in the GWAS dataset than any other set. This approach was used to analyze 150 different phenotypes obtained by principle component analysis of contrast maps from fMRI scanning of the Hariri faces task in 246 subjects. Significant hits were obtained with genes involved in blood function, GABA receptor function, mitochondrial pathways, and receptor clustering.

Introducing multi-modal analyses, Jingyu Liu described a novel parallel independent component analysis approach to understanding schizophrenia. Genotyping and fMRI datasets are analyzed simultaneously to identify the underlying independent components of the two datasets and subsequently the relationship between them (Liu et al., 2009). Liu et al. analyzed 5,000 SNPs selected because they showed loose association with the disease phenotype or were in genes previously implicated in the etiology of schizophrenia. The fMRI dataset was obtained from an auditory oddball task during which patients and controls press a button in response to atypical sounds. This approach identified groups of SNPs that were associated with specific components of the fMRI signal.

New computational methods for interpreting multi-modal MRI datasets were presented by Arvid Lundervold. Structural MRI, fMRI, and DTI data acquired from a subject in a single session are co-registered into the same spatial domain, allowing detailed analysis of structural and functional pathways within the brain. This approach can be used to model connectivity between different brain areas. On the same subject, Alireza Salami described the application of computational neuroimaging to address hippocampal function in age-related changes in episodic memory. Functional MRI imaging data from a face/name memory test were collected together with DTI and structural MRI data. Voxel-wise (univariate) and independent component (multivariate) analyses revealed a specific age-related decline in hippocampal function and an independent reduction in white matter integrity.

Moving on to collaborative approaches to analyzing complex traits, Jason Stein presented preliminary results from the ENIGMA consortium (Enhancing NeuroImaging Genetics through MetaAnalysis). GWAS and MRI scans are expensive to execute, making it difficult for single labs to collect suitably large datasets. ENIGMA is gathering GWAS and MRI data on a collaborative basis from over 90 members in 9 countries. The pilot project analyzed genetic determinants of hippocampal volume from meta-analysis of data from 8,000 subjects. No genome-wide significant hits were found, but the most strongly associated variant was identified independently by another consortium, strongly supporting collaborative approaches.

\section{FUNCTIONAL CORRELATES OF GENETIC VARIANTS}

The "holy grail" of complex trait analysis is to define functional correlates of risk alleles identified by association studies. For genetic variants implicated in cognitive and brain imaging traits, a major focus is on identifying their effect on brain function and their role in susceptibility to brain disorders such as psychiatric disorders or neurodegenerative diseases.

Sven Cichon explained how GWAS of schizophrenia and BPAD have yielded several replicated loci. For ZNF804A, fMRI analysis of a working memory task in healthy individuals showed that the risk allele is associated with altered connectivity in the dorsolateral prefrontal cortex (Esslinger et al., 2009). In a test of ability to attribute mental states to others, risk allele carriers had altered activity and connectivity in prefrontal and temporoparietal regions involved in social cognition (Walter et al., 2011). Risk alleles of NCAN, implicated in BPAD by GWAS (Cichon et al., 2011), were associated with reduced cortical area in schizophrenia patients and with differences in hippocampal activation during an episodic memory task in healthy subjects. Thus, imaging can delineate neural mechanisms for genetic variants identified in GWAS of psychiatric disorders.

Ole Andreassen presented correlations between genetics and brain imaging phenotypes in the Thematically Organized Psychosis (TOP) cohort of patients with severe psychiatric disorders. Candidate gene sets were tested for association with brain volume, cortical thickness, cortical area, and intracranial volume. Amongst genes implicated in microcephaly, variants in CDK5RAP2 and MECP2 were significantly associated with cortical area (Joyner et al., 2009; Rimol et al., 2010). A study of 10 genes involved in synaptic plasticity and formation of perineural nets showed that common B3GAT2 variants were associated with schizophrenia risk and cortical area (Kahler et al., 2011). Consistent with GWAS data, a significant association was found in schizophrenia patients between ventricle size and a SNP in the major histocompatibility cluster (Agartz et al., 2011). In a GWAS of schizophrenia, reduced cortical thickness was associated with SNPs in the $15 q 12$ region (Bakken et al., 2011).

Decline in episodic memory and decrease of hippocampal volume are accelerated in patients with Alzheimer's disease (AD). Beata Ferencz explained that although APOE4 is a well-known $\mathrm{AD}$ risk allele, there is growing evidence that TOMM40, adjacent to $A P O E$, is also implicated in neurodegeneration (Potkin et al., 2009). TOMM40 genotype did not affect free recall or hippocampal volume; however, hippocampal volume was positively correlated with episodic memory, and this was driven by the TOMM40 G allele in healthy individuals.

Janita Bralten described how AD risk alleles are associated with $\mathrm{AD}$-related structural brain changes in healthy individuals. When variants in genes previously identified in GWAS of AD were typed in over 900 healthy young people (Bralten et al., 2011), carriers of CR1 and SORL1 risk alleles had reduced volumes of the entorhinal cortex and hippocampus respectively, which may increase susceptibility to neurodegeneration. 
Michèle Wessa presented work on neuropsychological testing in three groups at high risk of BPAD: carriers of CACNA1C or $A N K 3$ risk alleles, first-degree relatives of BPAD patients, and individuals with hypomanic personality. In a task that assesses the effects of reward, punishment, and rule changes, all groups showed increased orbitofrontal activation in response to reward. $A N K 3$ risk variant carriers and first-degree relatives had disrupted DTI connectivity. Other neuropsychological tests revealed structural and functional changes in the high risk groups which could serve as endophenotypes for better diagnosis and understanding of BPAD.

Expression-based animal models have previously been used to identify candidate genes implicated in synaptic plasticity in rats. Le Hellard et al. (2009) identified significant association between genetic variants in one of these genes, DCLK1 (doublecortinand calmodulin kinase-like 1), and verbal memory, general cognition, and several psychiatric disorders (schizophrenia, BPAD, and attention deficit hyperactivity disorder, ADHD). This suggests a common effect of DCLK1 genetic variants on these phenotypes.

The importance of multi-disciplinary approaches to understand the prevalence, core symptoms, genetic risk factors, and neurological impact of persistent ADHD was emphasized by Jan Haavik. Genes recently implicated in ADHD susceptibility include LPHN3, CDH13, TPH1, and TPH2 (Arcos-Burgos et al., 2010; Halmoy et al., 2010). Because GWAS have proved relatively inefficient at finding variants with a clear functional effect at the molecular level, other strategies are being pursued in parallel, such as whole-exome sequencing.

To study major depressive disorder (MDD), James Cole investigated 17 genes that were down-regulated in a post-mortem microarray analysis of brains from MDD patients (Aston et al., 2005). White matter integrity was analyzed by DTI in 66 cases and 66 controls, and association studies were performed with 398 SNPs in the 17 genes. White matter integrity was significantly associated with variants in $L P A R 1 / E D G 1$, which encodes a protein involved in maintenance and formation of myelin.

\section{CONCLUSION AND FUTURE DIRECTIONS}

The aim of this Perspective is not to summarize all the advances in the field of imaging and cognition genetics but rather to present the major themes that emerged from the ICG 2011 meeting. Continued success in these three areas - identifying tractable phenotypes, applying new methods, and finding functional correlates of genetic variants - is critically dependent on intensively phenotyped cohorts. These are constantly growing in scale and scope.

\section{REFERENCES}

Agartz, I., Brown, A. A., Rimol, L. M., Hartberg, C. B., Dale, A. M., Melle, I., Djurovic, S., and Andreassen, O. A. (2011). Common sequence variants in the major histocompatibility complex region associate with cerebral ventricular size in schizophrenia. Biol. Psychiatry 70, 696-698.

Arcos-Burgos, M., Jain, M., Acosta, M. T., Shively, S., Stanescu, H., Wallis, D., Domene, S., Velez, J. I.,

Projects involving genetic association tests must have replication samples available at the outset. ICG 2011 provided an important forum for exchange of information about relevant samples. Because cognitive and imaging traits are probably influenced by multiple genetic variants of small effect, the consortium approach will assume greater importance by co-ordinating meta-analysis of data from thousands or tens of thousands or subjects. For all studies, quality control is essential at all stages of data collection and analysis, as is full reporting of the methods and parameters used.

In disciplines where technology, methods, and concepts are advancing rapidly, it is beneficial to meet regularly to discuss new developments and enhance collaborations so that all resources are used to best advantage. ICG 2011 has provided a firm foundation for future progress. A second meeting is scheduled for 22-23rd May 2013 in the same (very popular) location; please see www.icg2013.no for further information.

\section{ACKNOWLEDGMENTS}

We thank the conference speakers whose talks are summarized in this report: Ian Deary (University of Edinburgh, UK), Thomas Espeseth (University of Oslo, Norway), Yulia Kovas (Kings College London, UK), Andreas Papassotiropoulos (University of Basel, Switzerland), Kenneth Hugdahl (University of Bergen, Norway), Anders Fjell (University of Oslo, Norway), Lars Nyberg (University of Umeå, Sweden), Lorna Lopez (University of Edinburgh, UK), Gail Davies (University of Edinburgh, UK), Andrea Christoforou (University of Bergen, Norway), Kari Ersland (University of Bergen, Norway), Andrew Brown (University of Oslo, Norway), Jingyu Liu (Mind Research Network, Albuquerque, USA), Arvid Lundervold (University of Bergen, Norway), Alireza Salami (University of Umeå, Sweden), Jason Stein (University of California, Los Angeles, USA), Sven Cichon (Research Centre Juelich and University of Bonn, Germany), Ole Andreassen (University of Oslo, Norway), Beata Ferencz (Karolinska Institute, Sweden), Janita Bralten (Donders Institute, Nijmegen, Netherlands), Michèle Wessa (Central Institute of Mental Health, Mannheim, Germany), Jan Haavik (University of Bergen, Norway), and James Cole (King's College London, UK). We also gratefully acknowledge our colleagues who contributed to the meeting but whose presentations could not be summarized here due to space limitations. We thank the Bergen University Fond (http://www.uib.no/fond/) and the Norwegian Research School in Medical Imaging (http://www.ntnu.edu/medicalimaging) for their financial support.

Renner, T., Jacob, C., Lesch, K. P., Swanson, J., Vortmeyer, A., BaileyWilson, J. E., Castellanos, F. X., and Muenke, M. (2010). A common variant of the latrophilin 3 gene, LPHN3, confers susceptibility to ADHD and predicts effectiveness of stimulant medication. Mol. Psychiatry 15, 1053-1066.

Aston, C., Jiang, L., and Sokolov, B. P. (2005). Transcriptional profiling reveals evidence for signaling and oligodendroglial abnormalities in the temporal cortex from patients with major depressive disorder. Mol. Psychiatry 10, 309-322.

Bakken, T. E., Bloss, C. S., Roddey, J. C., Joyner, A. H., Rimol, L. M., Djurovic, S., Melle, I., Sundet, K., Agartz, I., Andreassen, O. A., Dale, A. M., and Schork, N. J. (2011). Association of genetic variants on $15 \mathrm{q} 12$ with cortical thickness and cognition in schizophrenia. Arch. Gen. Psychiatry 68, 781-790. 
Bralten, J., Arias-Vasquez, A., Makkinje, R., Veltman, J. A., Brunner, $\mathrm{H}$. G., Fernandez, G., Rijpkema, M., and Franke, B. (2011). Association of the Alzheimer's gene SORL1 with hippocampal volume in young, healthy adults. Am. J. Psychiatry 168, 1083-1089.

Chiang, M. C., McMahon, K. L., de Zubicaray, G. I., Martin, N. G., Hickie, I., Toga, A. W., Wright, M. J., and Thompson, P. M. (2011). Genetics of white matter development: a DTI study of 705 twins and their siblings aged 12 to 29 . Neuroimage 54 , 2308-2317.

Christoforou, A., Dondrup, M., Mattingsdal, M., Mattheisen, M., Giddaluru, S., Nothen, M. M., Rietschel, M., Cichon, S., Djurovic, S., Andreassen, O., Jonassen, I., Steen, V. M., Puntervoll, P., and Le Hellard, S. (2012). Linkagedisequilibrium-based binning affects interpretation of GWASs. Am. J. Hum. Genet. 90, 727-733.

Cichon, S., Muhleisen, T. W., Degenhardt, F. A., Mattheisen, M., Miro, X., Strohmaier, J., Steffens, M., Meesters, C., Herms, S., Weingarten, M., Priebe, L., Haenisch, B., Alexander, M., Vollmer, J., Breuer, R., Schmal, C., Tessmann, P., Moebus, S., Wichmann, H. E., Schreiber, S., MullerMyhsok, B., Lucae, S., Jamain, S., Leboyer, M., Bellivier, F., Etain, B., Henry, C., Kahn, J. P., Heath, S., Hamshere, M., O’Donovan, M. C., Owen, M. J., Craddock, N., Schwarz, M., Vedder, H., KammererCiernioch, J., Reif, A., Sasse, J., Bauer, M., Hautzinger, M., Wright, A., Mitchell, P. B., Schofield, P. R., Montgomery, G. W., Medland, S. E., Gordon, S. D., Martin, N. G., Gustafsson, O., Andreassen, O., Djurovic, S., Sigurdsson, E., Steinberg, S., Stefansson, H., Stefansson, K., Kapur-Pojskic, L., Oruc, L., Rivas, F., Mayoral, F., Chuchalin, A., Babadjanova, G., Tiganov, A. S., Pantelejeva, G., Abramova, L. I., Grigoroiu-Serbanescu, M., Diaconu, C. C., Czerski, P. M., Hauser, J., Zimmer, A., Lathrop, M., Schulze, T. G., Wienker, T. F., Schumacher, J., Maier, W., Propping, P., Rietschel, M., and Nothen, M. M. (2011). Genomewide association study identifies genetic variation in neurocan as a susceptibility factor for bipolar disorder. Am. J. Hum. Genet. 88, 372-381.

Davies, G., Tenesa, A., Payton, A., Yang, J., Harris, S. E., Liewald, D., Ke, X., Le, H. S., Christoforou, A., Luciano, M., McGhee, K., Lopez, L., Gow, A. J., Corley, J., Redmond, P., Fox,
H. C., Haggarty, P., Whalley, L. J., McNeill, G., Goddard, M. E., Espeseth, T., Lundervold, A. J., Reinvang, I., Pickles, A., Steen, V. M., Ollier, W., Porteous, D. J., Horan, M., Starr, J. M., Pendleton, N., Visscher, P. M., and Deary, I. J. (2011). Genomewide association studies establish that human intelligence is highly heritable and polygenic. Mol. Psychiatry 16, 996-1005.

de Quervain, D. J., Kolassa, I. T., Ertl, V., Onyut, P. L., Neuner, F., Elbert, T., and Papassotiropoulos, A. (2007). A deletion variant of the alpha2b-adrenoceptor is related to emotional memory in Europeans and Africans. Nat. Neurosci. 10, 1137-1139.

Deary, I. J., Penke, L., and Johnson, W. (2010). The neuroscience of human intelligence differences. Nat. Rev. Neurosci. 11, 201-211.

Docherty, S. J., Davis, O. S., Kovas, Y., Meaburn, E. L., Dale, P. S., Petrill, S. A., Schalkwyk, L. C., and Plomin, R. (2010). A genome-wide association study identifies multiple loci associated with mathematics ability and disability. Genes Brain Behav. 9, 234-247.

Docherty, S. J., Kovas, Y., and Plomin, R. (2011). Gene-environment interaction in the etiology of mathematical ability using SNP sets. Behav. Genet. 41, 141-154.

Espeseth, T., Endestad, T., Rootwelt, H., and Reinvang, I. (2007). Nicotine receptor gene CHRNA4 modulates early event-related potentials in auditory and visual oddball target detection tasks. Neuroscience 147, 974-985.

Espeseth, T., Sneve, M. H., Rootwelt, H., and Laeng, B. (2010). Nicotinic receptor gene CHRNA4 interacts with processing load in attention. PLoS ONE 5, e14407. doi:10.1371/journal.pone.0014407

Esslinger, C., Walter, H., Kirsch, P., Erk, S., Schnell, K., Arnold, C., Haddad, L., Mier, D., Opitz von, B. C., Raab, K., Witt, S. H., Rietschel, M. Cichon, S., and Meyer-Lindenberg, A. (2009). Neural mechanisms of a genome-wide supported psychosis variant. Science 324, 605.

Fjell, A. M., Westlye, L. T., Amlien, I., Espeseth, T., Reinvang, I., Raz, N., Agartz, I., Salat, D. H., Greve, D. N., Fischl, B., Dale, A. M., and Walhovd, K. B. (2009). High consistency of regional cortical thinning in aging across multiple samples. Cereb. Cortex 19, 2001-2012.

Halmoy, A., Johansson, S., Winge, I., McKinney, J. A., Knappskog, P. M., and Haavik, J. (2010).
Attention-deficit/hyperactivity disorder symptoms in offspring of mothers with impaired serotonin production. Arch. Gen. Psychiatry 67, 1033-1043.

Herold, C., Steffens, M., Brockschmidt, F. F., Baur, M. P., and Becker, T. (2009). INTERSNP: genome-wide interaction analysis guided by a priori information. Bioinformatics 25, 3275-3281.

Hugdahl, K. (2009). "Hearing voices": auditory hallucinations as failure of top-down control of bottom-up perceptual processes. Scand. J. Psychol. 50, 553-560.

Hugdahl, K., Loberg, E. M., and Nygard, M. (2009). Left temporal lobe structural and functional abnormality underlying auditory hallucinations in schizophrenia. Front. Neurosci. 3:34-45. doi:10.3389/neuro.01.001.2009

Joyner, A. H., CR, J., Bloss, C. S., Bakken, T. E., Rimol, L. M., Melle, I., Agartz, I., Djurovic, S., Topol, E. J., Schork, N. J., Andreassen, O. A. and Dale, A. M. (2009). A common MECP2 haplotype associates with reduced cortical surface area in humans in two independent populations. Proc. Natl. Acad. Sci. U.S.A. 106, 15483-15488.

Kahler, A. K., Djurovic, S., Rimol, L. M., Brown, A. A., Athanasiu, L., Jonsson, E. G., Hansen, T., Gustafsson, O., Hall, H., Giegling, I., Muglia, P., Cichon, S., Rietschel, M., Pietilainen, O. P., Peltonen, L., Bramon, E., Collier, D., St Clair, D., Sigurdsson, E. Petursson, H., Rujescu, D., Melle, I., Werge, T., Steen, V. M., Dale, A. M., Matthews, R. T., Agartz, I., and Andreassen, O. A. (2011). Candidate gene analysis of the human natural killer-1 carbohydrate pathway and perineuronal nets in schizophrenia: B3GAT2 is associated with disease risk and cortical surface area. Biol. Psychiatry 69, 90-96.

Le Hellard, S., Havik, B., Espeseth, T., Breilid, H., Lovlie, R., Luciano, M. Gow, A. J., Harris, S. E., Starr, J. M., Wibrand, K., Lundervold, A. J., Porteous, D. J., Bramham, C. R. Deary, I. J., Reinvang, I., and Steen, V. M. (2009). Variants in doublecortinand calmodulin kinase like 1, a gene up-regulated by BDNF, are associated with memory and general cognitive abilities. PLoS ONE 4, e7534. doi:10.1371/journal.pone.0007534

Lind, J., Persson, J., Ingvar, M., Larsson, A., Cruts, M., Van, B. C., Adolfsson, R., Backman, L., Nilsson, L. G., Petersson, K. M., and Nyberg, L. (2006). Reduced functional brain activity response in cognitively intact apolipoprotein E epsilon 4 carriers. Brain 129, 1240-1248.

Liu, J., Pearlson, G., Windemuth, A., Ruano, G., Perrone-Bizzozero, N. I., and Calhoun, V. (2009). Combining fMRI and SNP data to investigate connections between brain function and genetics using parallel ICA. Hum. Brain Mapp. 30, 241-255.

Penke, L., Munoz, M. S., Murray, C., Gow, A. J., Hernandez, M. C., Clayden, J. D., Starr, J. M., Wardlaw, J. M., Bastin, M. E., and Deary, I. J. (2010). A general factor of brain white matter integrity predicts information processing speed in healthy older people. J. Neurosci. 30, 7569-7574.

Persson, J., Lind, J., Larsson, A., Ingvar, M., Cruts, M., Van, B. C., Adolfsson, R., Nilsson, L. G., and Nyberg, L. (2006). Altered brain white matter integrity in healthy carriers of the APOE epsilon4 allele: a risk for $\mathrm{AD}$ ? Neurology 66, 1029-1033.

Potkin, S. G., Guffanti, G., Lakatos, A., Turner, J. A., Kruggel, F., Fallon, J. H., Saykin, A. J., Orro, A., Lupoli, S., Salvi, E., Weiner, M., and Macciardi, F. (2009). Hippocampal atrophy as a quantitative trait in a genome-wide association study identifying novel susceptibility genes for Alzheimer's disease. PLoS ONE 4, e6501. doi:10.1371/journal.pone.0006501

Rasch, B., Spalek, K., Buholzer, S., Luechinger, R., Boesiger, P., Papassotiropoulos, A., and de Quervain, D. J. (2009). A genetic variation of the noradrenergic system is related to differential amygdala activation during encoding of emotional memories. Proc. Natl. Acad. Sci. U.S.A. 106, 19191-19196.

Rimol, L. M., Agartz, I., Djurovic, S., Brown, A. A., Roddey, J. C., Kahler, A. K., Mattingsdal, M., Athanasiu, L., Joyner, A. H., Schork, N. J., Halgren, E., Sundet, K., Melle, I., Dale, A. M., and Andreassen, O. A. (2010). Sexdependent association of common variants of microcephaly genes with brain structure. Proc. Natl. Acad. Sci. U.S.A. 107, 384-388.

Stansberg, C., Ersland, K. M., van, d. V., and Steen, V. M. (2011) Gene expression in the rat brain: high similarity but unique differences between frontomedial-, temporal- and occipital cortex. BMC Neurosci. 12, 15. doi:10.1186/1471-2202-12-15

Walter, H., Schnell, K., Erk, S., Arnold, C., Kirsch, P., Esslinger, C., Mier, D., Schmitgen, M. M., Rietschel, M., Witt, S. H., Nothen, M. M., Cichon,S., and Meyer-Lindenberg, 
A. (2011). Effects of a genome-wide supported psychosis risk variant on neural activation during a theoryof-mind task. Mol. Psychiatry 16, 462-470.

Conflict of Interest Statement: The authors declare that the research was conducted in the absence of any commercial or financial relationships that could be construed as a potential conflict of interest.

Received: 21 March 2012; accepted: 03 May 2012; published online: 24 May 2012.
Citation: Le Hellard S and Hanson I (2012) The imaging and cognition genetics conference 2011, ICG 2011: a meeting of minds. Front. Neurosci. 6:74. doi: 10.3389/fnins.2012.00074

This article was submitted to Frontiers in Neurogenomics, a specialty of Frontiers in Neuroscience.
Copyright (C) 2012 Le Hellard and Hanson. This is an open-access article distributed under the terms of the Creative Commons Attribution Non Commercial License, which permits noncommercial use, distribution, and reproduction in other forums, provided the original authors and source are credited. 